\title{
Efficacy of Long-Term Treatment with Low-Dose Thalidomide for Patients with Relapsed/Refractory Multiple Myeloma
}

\author{
Kazuyuki Shimizu ${ }^{1}$, Hirokazu Murakami ${ }^{2}$, Morio Sawamura ${ }^{3}$, Yutaka Hattori ${ }^{4}$, Shinichiro Okamoto ${ }^{5}$, \\ Akiyoshi Miwa ${ }^{6}$, Isamu Sugiura ${ }^{7}$, Chihiro Shimazaki ${ }^{8}$, Masafumi Taniwaki ${ }^{8}$, Tadao Ishida ${ }^{9}$, Toshiaki \\ Hayashi $^{9}$, Hiroshi Kosugi ${ }^{10}$, Masaaki Yuge ${ }^{10}$, Shinsuke Iida ${ }^{11}$, Takashi Ishida ${ }^{11}$, Kazutaka Sunami ${ }^{12}$, \\ Hideki Asaoku $^{13}$, Akira Sakai ${ }^{14}$, Masahiro Abe ${ }^{15}$, Toshiyuki Takagi ${ }^{16}$
}

\begin{abstract}
${ }^{1}$ Department of Multimodal Therapy for Multiple Myeloma, Aichi Gakuin University School of Dentistry, Nagoya, Japan; ${ }^{2}$ Course of Health Sciences, Gunma University Graduate School of Health Sciences, Maebashi, Japan; ${ }^{3}$ Nishi-Gunma Hospital, Shibukawa, Japan; ${ }^{4}$ Division of Pharmacy, Keio University School of Medicine, Tokyo, Japan; ${ }^{5}$ Division of Hematology, Keio University School of Medicine, Tokyo, Japan; ${ }^{6}$ National Center for Global Health and Medicine, Tokyo, Japan; ${ }^{7}$ Toyohashi Municipal Hospital, Toyohashi, Japan; ${ }^{8}$ Kyoto Prefectural University of Medicine, Kyoto, Japan; ${ }^{9}$ Sapporo Medical University, Sapporo, Japan; ${ }^{10}$ Ogaki Municipal Hospital, Ogaki, Japan; ${ }^{11}$ Department of Medical Oncology and Immunology, Nagoya City University, Nagoya, Japan; ${ }^{12}$ Okayama Medical Center, Okayama, Japan; ${ }^{13}$ Hiroshima Red Cross Hospital, Hiroshima, Japan; ${ }^{14}$ Department of Hematology and Oncology, Hiroshima University, Hiroshima, Japan; ${ }^{15}$ Deaprtment of Medicine and Bioregulatory Sciences, Tokushima University, Tokushima, Japan; ${ }^{16}$ Kimitsu Chuou Hospital, Kisarazu, Japan.

Email: hmura@gunma-u.ac.jp
\end{abstract}

Received August 11 $1^{\text {th }}, 2011$; revised September $20^{\text {th }}, 2011$; accepted October $14^{\text {th }}, 2011$.

\begin{abstract}
Introduction: We report the results of a prospective study of long-tern treatment with single-agent thalidomide in patients who had responded in a preceding trial of the use of thalidomide for relapsed/refractory myeloma. Patients and Methods: Nineteen patients were enrolled: 11 patients (57.9\%) treated at a dosage of $100 \mathrm{mg} /$ day; 2 patients (10.5\%) at a dosage of $200 \mathrm{mg} /$ day; 2 patients (10.5\%) at a dosage of $300 \mathrm{mg} /$ day; and 4 patients (21.1\%) at a dosage of 400 $\mathrm{mg} /$ day. The median follow-up from the start of the preceding study was 3.0 years. At the time of entry to this study, 5 patients (26.3\%) had partial response (PR), another 5 patients (26.3\%) had a minimal response (MR), and the remaining 9 patients (47.4\%) had shown no change (NC). Results: The cumulative MR rate was $78.9 \%$ (at the $32^{\text {nd }}$ week) and the cumulative PR rate was $47.4 \%$ (at the $112^{\text {th }}$ week). The median progression-free survival was 104 weeks and the median time to next treatment was 144 weeks. No patients experienced grade 4 or greater hematologic toxicity or grade 3 or greater non-hematologic toxicity. Conclusion: Long-term thalidomide maintenance therapy induced an increase in response rate, suppressed the progression to active myeloma without severe adverse events, and contributed to long survival with good activities of daily living.
\end{abstract}

Keywords: Maintenance Therapy, Progression-Free Survival, Time to Next Treatment, Efficacy, Safety

\section{Introduction}

Whereas the positive role of thalidomide as a consolidation treatment after high-dose therapy with autologous stem cell transplantation (HDT-ASCT), in the context of newly diagnosed myeloma, has been clarified in the patients without high-risk cytogenetics who have obtained less than a very good partial response [1,2], its role as a maintenance therapy remains controversial because of its toxicity and the concern of potential induction of resistance to subsequent treatment [3-5]. In addition, we cur- rently have much less information on the role of thalidomide as maintenance therapy, in the context of cases of relapsed/refractory multiple myeloma, after successful salvage treatment. Based on the results of phase II trials in which the cumulative dose of thalidomide did not have an impact on the efficacy of maintenance therapy and toxicity increased above a dosage of $200 \mathrm{mg} / \mathrm{d}$ [6,7], treatment with low-dose thalidomide is now the preferred option.

We have conducted a prospective study to evaluate the efficacy and safety of thalidomide given as a single-agent maintenance therapy to patients with relapsed and/or re- 
fractory multiple myeloma who had been enrolled in a previous phase II study [8] and who achieved at least no change (NC) with thalidomide treatment as per the study protocol.

\section{Patients and Methods}

\subsection{Eligibility}

Patients were deemed eligible for enrollment in this study if they had responded and maintained at least $\mathrm{NC}$ assessed at the $16^{\text {th }}$ week of the phase II study period with single-agent thalidomide treatment given for at least 4 weeks [8]. According to the phase II study protocol, patients who achieved at least a minimal response (MR) continued on thalidomide treatment at the dosage with which the response had been obtained until the cutoff of the study. Otherwise, the dose of thalidomide was escalated by $100 \mathrm{mg}$ every 4 weeks until the cutoff of the study $\left(16^{\text {th }}\right.$ week) to a maximum of $400 \mathrm{mg} / \mathrm{d}$. In total, 19 patients had achieved and maintained a response of at least NC according to the European Group for Blood and Marrow Transplantation response criteria [9] by the cutoff of the phase II study and were studied.

All patients gave written informed consent and agreed to abide by strict contraception. The study and the written informed consent form were approved by the institutional review board of each participating hospital. The study was conducted in accordance with the Good Clinical Practice for Trials of Drugs and the Declaration of Helsinki.

\subsection{Treatment Schedule}

Single-agent thalidomide treatment was continued in the 19 patients at their individual final doses of the phase II study until disease progression or intolerance occurred, for a maximum of 3 years. Patients were evaluated every 4 weeks for response and drug toxicity. Thalidomide was supplied by the Fujimoto Pharmaceutical Corporation (Osaka, Japan) and was given orally before sleep. No antithrombotic prophylaxis was instituted because no patients experienced thromboembolic events during the phase II study.

\subsection{Response, Progression-Free Survival, Time to Next Ttreatment, and Toxicity Criteria}

Responses were assessed by the decrease in the monoclonal protein measured at the time of entry into the phase II study using the European Group for Blood and Marrow Transplantation response criteria [9]. Progressionfree survival (PFS) was measured from the date of initiation of thalidomide treatment in the phase II study until death or disease progression, whichever was earlier. Time to next treatment (TTNT) was measured from the date of initia- tion of thalidomide treatment until death or the date of initiation of the next treatment. The PFS and TTNT curves were constructed according to the Kaplan-Meier method. Toxicities were graded using the National Cancer Institute Common Toxicity criteria (version 3).

\section{Results}

\subsection{Patient Characteristics}

A total of 19 patients were enrolled between December 2005 and April 2006. Patients were followed until March 2009 and the median follow-up from the start of the phase II study was 3 years (156 weeks; range, 28 - 180 weeks). The characteristics of the 19 patients are shown in Table 1. The mean age was 60 years (range, 42 - 81 years). More than half of the patients had relapsed after HDT-ASCT.

Eleven patients (57.9\%) were treated with thalidomide at a dosage of $100 \mathrm{mg} /$ day, 2 patients $(10.5 \%)$ were treated at a dosage of $200 \mathrm{mg} /$ day, 2 patients $(10.5 \%)$ were treated at a dosage of $300 \mathrm{mg} /$ day, and 4 patients $(21.1 \%)$ were treated at a dosage of $400 \mathrm{mg} /$ day.

\subsection{Response}

At the time of entry to this study, 5 patients $(26.3 \%)$ had partial response (PR), another 5 patients $(26.3 \%)$ had MR,

Table 1. Patients characteristics.

\begin{tabular}{lc}
\hline Variables & Total \\
\hline Number of cases & 19 \\
Mean age (yr) & 60.0 \\
Range (yr) & $42-81$ \\
Time sinceDx(yr) & 5.03 \\
Range (yr) & $0.17-17$ \\
Sex (male/female) & $8 / 11$ \\
M protein type & \\
IgG & 12 \\
IgA & 6 \\
Light chain & 1 \\
PS (0/1/2) & $15 / 3 / 1$ \\
ISS stage (I/II/III) & $11 / 3 / 5$ \\
Prior therapy & \\
Chemotherapy & 8 \\
Lines (median, range) & $1.5,1-3$ \\
ASCT & 11 \\
$\beta$ 2M (mg/L) median, range & $2.50,1.0-10.24$ \\
LDH (IU/L) median, range & $105-346$ \\
\hline Dx; diagno, PS; perf &
\end{tabular}

Dx; diagnosis, PS; performance status, $\beta 2 \mathrm{M} ; \beta 2$ microgobulin. 
and the remaining 9 patients (47.4\%) had shown $\mathrm{NC}$ to the latest thalidomide therapy.

The cumulative response rate is shown in Figure 1. The reduction of M-protein was continued at the $112^{\text {th }}$ week. MR was obtained in $73.7 \%$ at the $24^{\text {th }}$ week and in $78.9 \%$ at the $32^{\text {nd }}$ week. PR was obtained $31.6 \%$ at the $24^{\text {th }}$ week, $31.6 \%$ at the $32^{\text {nd }}$ week, $42.1 \%$ at the $48^{\text {th }}$ week, and $47.4 \%$ at the $112^{\text {th }}$ week.

\subsection{Progression-Free Survival}

Figure 2 shows the PFS curve of the 19 patients studied; the median PFS was 104 weeks (1.99 years). The PFS rates were $73.0 \%$ at 1 year, $55.1 \%$ at 2 years, and $28.0 \%$ at 3 years. Only 1 patient has died on the $31^{\text {st }}$ week.

\subsection{Time to Next Treatment}

Figure 3 shows the TTNT curve of the 19 patients. The median TTNT was 144 weeks ( 2.76 years). The TTNT rate was $73.7 \%$ at 1 year, $63.2 \%$ at 2 years, and $47.4 \%$ at 3 years. Thalidomide/high-dose dexamethasone treatment was conducted in 6 patients and melphalan/prednisolone/thalidomide treatment was conducted in 3 patients after the discontinuance of single-agent thalidomide maintenance therapy. Eight patients continued single-dose thalidomide treatment over 40 months after progressive disease. Thalidomide was discontinued in only 2 patients.

\subsection{Toxicities}

All 19 patients experienced at least Grade 1 toxicity; however, no patient experienced Grade 4 or greater hema-

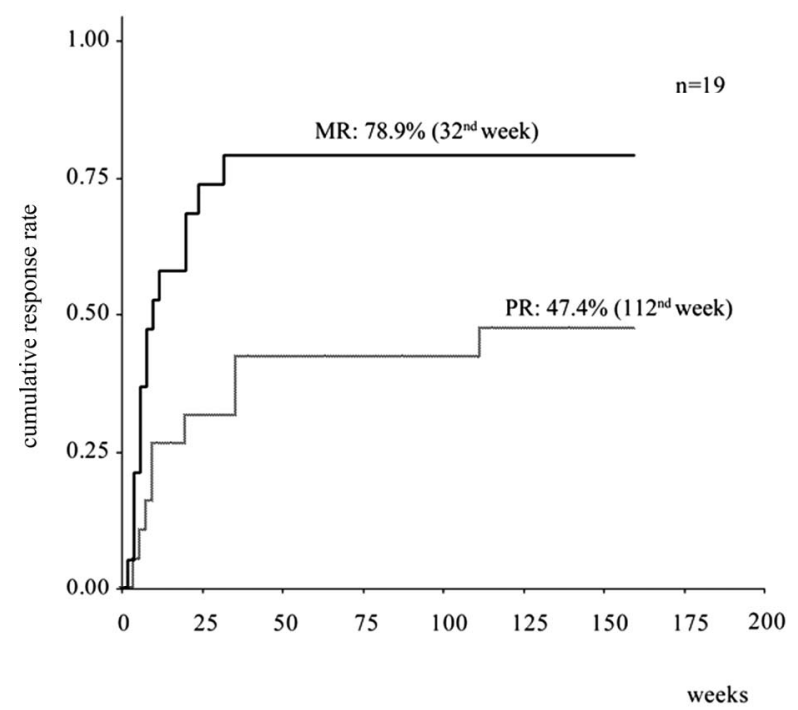

Figure 1. The cumulative response rate in patients treated with long-term thalidomide maintenance. The black line shows minimal response rate and the gray line shows partial response rate.

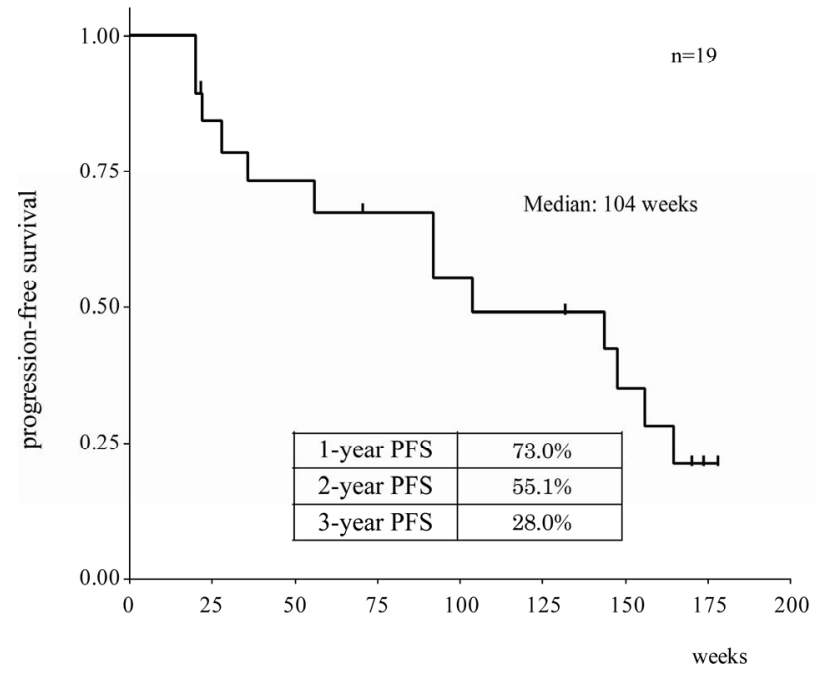

Figure 2. Progression-free survival in patients treated with long-term thalidomide maintenance. The curve was constructed according to the Kaplan-Meier method.

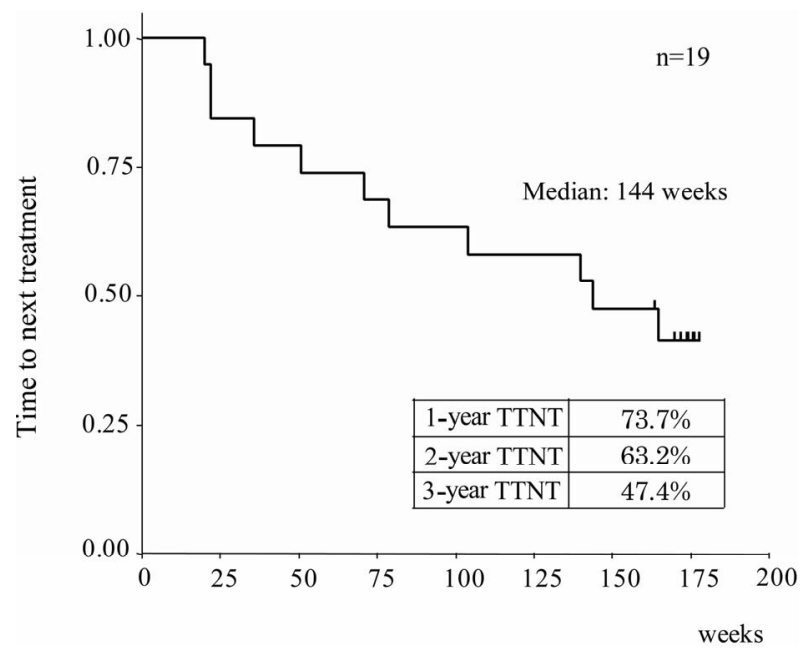

Figure 3. Time to next treatment in patients treated with long-term thalidomide maintenance. The curve was constructed according to the Kaplan-Meier method.

tologic toxicity or Grade 3 or greater non-hematologic toxicity. The toxicity profile observed in at least 10 patients is shown in Table 2. The most common Grade 3 hematologic toxicities were neutropenia in 9 patients $(47.4 \%)$, lymphopenia in $5(26.3 \%)$, and leucopenia in 4 (21.1\%). The most common Grade 2 non-hematologic toxicities were peripheral neuropathy in 3 patients $(15.8 \%)$, constipation in $2(10.5 \%)$, and skin rash in $2(10.5 \%)$. One patient receiving $200 \mathrm{mg} / \mathrm{d}$ thalidomide but no thromboprophylaxis experienced deep vein thrombosis on the $71^{\text {st }}$ week.

Toxicity or intolerance that resulted in the discontinuation or dose reduction of thalidomide occurred in 4 of 
Table 2. Toxicity profile.

\begin{tabular}{|c|c|c|c|c|}
\hline \multirow[b]{2}{*}{ Toxicity } & \multicolumn{4}{|c|}{$\mathrm{n}(\%)$} \\
\hline & Total & Grade 1 & Grade 2 & Grade 3 \\
\hline \multicolumn{5}{|l|}{ Hematological } \\
\hline Neutropeni & $14(73.7)$ & $1(5.3)$ & $4(21.1)$ & $9(47.4)$ \\
\hline Lymphopenia & $10(52.6)$ & $1(5.3)$ & $4(21.1)$ & $5(26.3)$ \\
\hline Leucopenia & $10(52.6)$ & $2(10.5)$ & $4(21.1)$ & $4(21.1)$ \\
\hline Basophilia & $11(57.9)$ & $11(57.9)$ & 0 & 0 \\
\hline \multicolumn{5}{|l|}{ Non-hematological } \\
\hline Constipation & $16(84.2)$ & $14(73.7)$ & $2(10.5)$ & 0 \\
\hline Peripheral neuropathy & $15(78.9)$ & $12(63.2)$ & $3(15.8)$ & 0 \\
\hline Somnolence & $14(73.7)$ & $13(68.4)$ & $1(5.3)$ & 0 \\
\hline Dry mouth & $11(57.9)$ & $11(57.9)$ & 0 & 0 \\
\hline Edema & $10(52.6)$ & $10(52.6)$ & 0 & 0 \\
\hline Tremor & $10(52.6)$ & $10(52.6$ & 0 & 0 \\
\hline Skin rash & $10(52.6)$ & $8(42.1)$ & $2(10.5)$ & 0 \\
\hline
\end{tabular}

the 19 patients $(21.1 \%)$. These 4 patients were treated with thalidomide at a dosage of over $200 \mathrm{mg} /$ day. Thalidomide treatment was discontinued in 2 patients: deep vein thrombosis occurred in 1 patient treated with 200 $\mathrm{mg} / \mathrm{d}$ on the $71^{\text {st }}$ week, and nephrotic syndrome occurred in 1 patient on the $132^{\text {nd }}$ week. Thalidomide dosage was reduced in another 2 patients because of peripheral neuropathy and neutropenia. No patients treated with 100 $\mathrm{mg} / \mathrm{d}$ thalidomide discontinued treatment due to toxicity.

\section{Discussion}

This study was conducted prospectively to evaluate the efficacy and safety of long-term treatment with singleagent thalidomide in patients who had been enrolled and obtained at least NC in a phase II trial of the use of thalidomide in patients with relapsed/refractory multiple myeloma [8]. The dosage of thalidomide in this continuous treatment study was determined by the one with which each patient had responded by achieving at least NC in the preceding phase II trial.

Nineteen patients were enrolled. Eleven patients (57.9\%) were treated with continuous thalidomide treatment at a dosage of $100 \mathrm{mg} /$ day, 2 patients $(10.5 \%)$ were treated at a dosage of $200 \mathrm{mg} /$ day, 2 patients $(10.5 \%)$ were treated at a dosage of $300 \mathrm{mg} / \mathrm{day}$, and 4 patients $(21.1 \%)$ were treated at a dosage of $400 \mathrm{mg} /$ day. The discontinuation or dose reduction of thalidomide occurred in 4 patients
(21.1\%). These 4 patients were treated with thalidomide at a dosage of over $200 \mathrm{mg} /$ day. In contrast, no patients treated with $100 \mathrm{mg} / \mathrm{d}$ discontinued thalidomide due to toxicity. According to these findings, low-dose thalidomide might be adequate for the maintenance treatment for multiple myeloma.

The beneficial effect of long-term treatment with thalidomide was observed at least until week $112(2.15$ years). The PR rate was $26.3 \%$ at the time of initiation of maintenance treatment ( $16^{\text {th }}$ week) and continuously increased up to $47.4 \%$ at the $112^{\text {th }}$ week. The MR rate also increased from $26.3 \%$ at the initiation of maintenance treatment to $78.9 \%$ at the $32^{\text {nd }}$ week. Singhal et al. reported that the response of thalidomide treatment was obtained within 4 months in patients with refractory myeloma [10]; however, our study revealed the response of long-term thalidomide maintenance treatment gradually increased over 4 months after initiation of thalidomide.

The PFS was fairly long in this study. It is difficult to compare this result with those in the published reports on single-agent thalidomide treatment in cases of relapsed/ refractory multiple myeloma, because most trials used a starting thalidomide dose of $200 \mathrm{mg} / \mathrm{d}$ and utilized a dose escalation up to $800 \mathrm{mg} / \mathrm{d}$, and also because the followup period is not as long as that in our study $[6,11]$. The case series studied may be mostly composed of low-risk patients in terms of age, International Staging System stages, 
$\beta 2$-microglobulin levels, and lactate dehydrogenase levels. Recent studies have shown the importance of obtaining complete response (CR) not only in patients with newly diagnosed multiple myeloma but also relapsed/refractory multiple myeloma $[12,13]$. However, in patients in the low-risk category, survival is not significantly different between patients with CR and those with PR [14]. Another recent study has disclosed that maintaining CR is more important than obtaining CR in terms of longer survival duration [15]. It is also noted that patients with low-risk disease can survive longer with PR status [14]. In our patients, long-term maintenance treatment with thalidomide upgraded the initial response status and sustained the upgraded response status, which resulted in prolonged PFS.

The TTNT was extremely long compared with the PFS. Eight patients continued single-dose thalidomide treatment after progressive disease because of the absence of progression to active myeloma, namely clinical relapse [16]. According to this finding, one of the reasons for the long TTNT might depend on the slow progression to active myeloma during thalidomide maintenance treatment. Our results are in agreement with the comment of Stewart [17] that, in a slower-tempo relapse, sequencing of drugs may offer superior overall survival results.

With regard to a long-term treatment with thalidomide, there has been a concern of the late development of neuropathy if given sufficient length of time with low-dose thalidomide [18]. However, long-term treatment with low-dose thalidomide for as long as 3 years in the present study did not result in delayed development of adverse events. Furthermore, because of the lower toxicity of low-dose thalidomide, the patients could stay on the treatment and enjoyed a long-term survival with good activities of daily living.

\section{Acknowledgements}

We thank the patients who agreed to participate in this study.

\section{REFERENCES}

[1] M. Attal, J. L. Harousseau, S. Leyvraz, C. Doyen, C. Hulin, L. Benboubker, I. Y. Agha, J.-H. Bourhis, L. Garderet, B. Pegourie, C. Dumontet, M. Renaud, L. Voillat, C. Berthou, G. Marit, M. Monconduit, D. Caillot, B. Grobois, H. Avet-Loiseau, P. Moreau and T. Facon, "Maintenance Therapy with Thalidomide Improves Survival in Patients with Multiple Myeloma," Blood, Vol. 108, No. 10, 2006, pp. 3289-3294. doi:10.1182/blood-2006-05-022962

[2] A. Spencer, H. Miles-Prince, A. W. Roberts, I. W. Prosser, K. F. Bradstock, L. Coyle, D. S. Gill, N. Horvath, J. Reynolds and N. Kennedy, "Consolidation Therapy with Low-Dose Thalidomide and Prednisolone Prolongs the
Survival of Multiple Myeloma Patients Undergoing a Single Autologous Stem-Cell Transplantation Procedure," Journal of Clinical Oncology, Vol. 27, No. 11, 2009, pp. 1788-1793. doi:10.1200/JCO.2008.18.8573

[3] B. Barlogie, G. Tricot, E. Anaissie, J. Shaughnessy, E. Rasmussen, F. van Rhee, A. Fassas, M. Zangari, K. Hollmig, M. Pineda-Roman, C. Lee, G. Talamo, R. Thertulien, E. Kiwan, S. Krishna, M. Fox and J. Crowley, "Thalidomide and Hematopoietic-Cell Transplantation for Multiple Myeloma," The New England Journal of Medicine, Vol. 354, No. 10, 2006, pp. 1021-1030. doi:10.1056/NEJMoa053583

[4] G. J. Morgan, F. E. Davies, W. M. Gregory, S. E. Bell, A J. Szubert, K. Cocks, N. N. Coy, M. T. Drayson, R. G. Owen, F. M. Ross, G. H. Jackson and J. A. Child, "The Addition of Thalidomide to the Induction Treatment of Newly Presenting Myeloma Patients Increase the CR Rate Which Is Likely to Translate into Improved PFS and OS," Blood, Vol. 114, No. 22, 2009, p. 114.

[5] H. M. Lokhorst, .B van der Holt, S. Zweegman, E. Vellenga, S. Croockewit, M. H. van Oers, P. von dem Borne, P. Wijermans, R. Schaafsma, O. de Weerdt, S. Wittebol, M. Delforge, H. Berenschot, G. M. Bos, K.-S. G. Jie, H. Sinnige, M. van Marwijk-Kooy, P. Joosten, M. C. Minnema, R. van Ammerlaan and P. Sonneveld, "A Randomized Phase III Study on the Effect of Thalidomide Combined with Adriamycin, Dexamethasone (TAD), and High-Dose Melphalan, Followed by Thalidomide Maintenance in Patients with Multiple Myeloma," Blood, Vol. 115, No. 6, 2010, pp. 1113-1120. doi:10.1182/blood-2009-05-222539

[6] A. Glasmacher, C. Hahn, F. Hoffmann, R. Neumann, H. Goldschmidt, M. von Lilienfeld-Toal, K. Orlopp, I. Schmidt-Wolf and M. Gorschlüter, "A Systematic Review of Phase-II Trials of Thalidomide Monotherapy in Patients with Relapsed or Refractory Multiple Mye- loma," British Journal of Haematology, Vol. 132, No. 5, 2006, pp. 584-593. doi:10.1111/j.1365-2141.2005.05914.X

[7] S. Feyler, A. Rawstron, G. Jackson, J. A. Snowdon, K. Cocks and R. J. Johnson, "Thalidomide Maintenance Following High-Dose Therapy in Multiple Myeloma: A UK Myeloma Forum Phase II Study," British Journal of Haematology, Vol. 139, No. 3, 2007, pp. 429-433. doi:10.1111/j.1365-2141.2007.06817.x

[8] H. Murakami, K. Shimizu, M. Sawamura, K. Suzuki, I. Sugiura, H. Kosugi, C. Shimazaki, M. Taniwaki, M. Abe, and T. Takagi, "Phase II and Pharmacokinetic Study of Thalidomide in Japanese Patients with Relapsed/Refractory Multiplemyeloma," International Journal of Hematology, Vol. 89, No. 5, 2009, pp. 636-641. doi:10.1007/s12185-009-0314-5

[9] J. Bladé, D. Samson, D. Reece, J. Apperley, B. Björkstrand, G. Gahrton, M. Gertz, S. Giralt, S. Jagannath and D. Vesole, "Criteria for Evaluating Disease Response and Progression in Patients with Multiple Myeloma Treated by High-Dose Therapy and Haemopoietic Stem Cell Transplantation," British Journal of Haematology, Vol. 102, No. 5, 1998, pp. 1115-1123. doi:10.1046/j.1365-2141.1998.00930.x 
[10] S. Singhal, J. Mehta, R. Desikan, D. Ayers, P. Roberrson, P. Eddlemon, N. Munshi, E. Anaissie, C. Wilson, M. Dhodapkar, J. Zeldis and B. Barlogie, "Antitumor Activity of Thalidomide in Refractory Multiple Myeloma," The New England Journal of Medicine, Vol. 341, No. 21, 1999, pp. 1565-1571. doi:10.1056/NEJM199911183412102

[11] H. M. Prince, B. Schenkel and L. Mileshkin, "An Analysis of Clinical Trials Assessing the Efficacy and Safety of Single-Agent Thalidomide in Patients with Relapsed or Refracttory Multiple Myeloma," Leukemia \& Lymphoma, Vol. 48, No. 1, 2007, pp. 46-55. doi:10.1080/10428190601001904

[12] A. Corso, P. Zappasodi, L. Barbarano, M. T. Petrucci, A. Palumbo, T. Caravita, S. Mangiacavalli, A. M. Cafro, M. Varettoni, F. Gay, E. Morra and M. Lazzarino, "LongTerm Outcome in Relapsed and Refractory Multiple Myeloma Treated with Thalidomide. Balancing Efficacy and Side-Effects," Leukemia Research, Vol. 33, No. 9, 2009, pp. 145-149. doi:10.1016/j.leukres.2009.03.015

[13] A. A. Chanan-Khan and S. Giralt, "Importance of Achieving a Complete Response in Multiple Myeloma and the Impact of Novel Agents," Journal of Clinical Oncology, Vol. 28, No. 15, 2010, pp. 2612-2624. doi:10.1200/JCO.2009.25.4250

[14] J. Haessler, J. D. Shaughnessy, F. Zhan, J. Crowley, J. Epstein, F. van Rhee, E. Anaissie, M. Pineda-Roman, M. Zangari, K. Hollmig, A. Mohiuddin, Y. Alsayed, A. Hoering, G. Tricot and B. Barlogie, "Benefit of Complete re- sponse in Multiple Myeloma Limited to High-Risk Subgroup Identified by Gene Expression Profiling," Clinical Cancer Research, Vol. 13, No. 23, 2007, pp. 7073-7079. doi:10.1158/1078-0432.CCR-07-0527

[15] B. Barlogie, E. Anaissie, J. Haessler, F. van Rhee, M. Pineda-Roman, K. Hollmig, Y. Alsayed, J. Epstein, J. D. Shaughnessy Jr. and J. Crowley, "Complete Remission Sustained 3 Years from Treatment Initiation Is a Powerful Surrogate for Extended Survival in Multiple Myeloma," Cancer, Vol. 113, No. 2, 2008, pp. 355-359. doi:10.1002/cncr.23546

[16] B. G. Durie, J. L. Harousseau, S. J. Miguel, J. Blade, B. Barlogie, K. Anderson, M. Gertz, M. Dimopoulos, J. Westin, P. Sonneveld, H. Ludwig, G. Gahrton, M. Beksac, J. Crowley, A. Belch, M. Boccadaro, M. Cavo, I. Turesson, D. Joshua, D. Vesole, R. Kyle, R. Alexanian, G. Tricot, M. Attal, G. Merlini, R. Powles, P. Richardson, K. Shimizu, P. Tosi, G. Morgan and S. V. Rajkumar, "International Uniform Response Criteria for Multiple Myeloma," Leukemia, Vol. 20, No. 9, 2006, pp. 1467-1473. doi:10.1038/sj.leu.2404284

[17] A. K. Stewart, "Novel Therapies for Relapsed Myeloma," Hematology 2009 American Society of Hematology Education Program, Scottsdale, 2009, pp. 578-586.

[18] L. Mileshkin and H. M. Prince, "The Troublesome Toxicity of Peripheral Neuropathy with Thalidomide," Leukemia \& Lymphoma, Vol. 47, No. 11, 2006, pp. 22762279. doi:10.1080/10428190600948303 